\title{
Internal sacrococcygeal teratoma causing urinary retention in a newborn
}

\begin{abstract}
A-9-day old term female neonate presented with urinary retention secondary to an enlarging cystic pelvic mass.MRI showed a huge presacralcystic mass which pushed the rectum and uterus anteriorly and the bowels superiorly. Excision of the cystic mass and removal of the coccyx (coccygectomy) were performed. Her urinary retention improved after surgery. We report an uncommon case of Type IV Sacrococcygeal teratoma (SCT) causing urinary retention in a newborn.
\end{abstract}

Keywords: neonate, urinary retention, pelvic mass, sct, sacrococcygeal teratoma, afp, alpha-fetoprotein

\author{
Volume 6 Issue 5 - 2017 \\ Arief Hamid,' Zarina A Latiff, ${ }^{2}$ Dayang A Aziz ${ }^{3}$ \\ 'Department of Surgery, University Sains Islam Malaysia, Malaysia \\ 2Department of Paediatrics, University Kebangsaan Malaysia \\ Medical Centre, Malaysia \\ ${ }^{3}$ Department of Surgery, University Kebangsaan Malaysia Medical \\ Centre, Malaysia
}

\begin{abstract}
Correspondence: Dayang Anita Abdul Aziz, Department of Surgery, UKM Medical Centre, Jalan Yaacob Latif, Cheras, 56000 Kuala Lumpur, Malaysia, Tel 6012305 9439, Fax 603 91456684, Email dayanganita@gmail.com
\end{abstract}

Received: December 20, 2015 | Published: April 19, 2017

\section{Introduction}

Cyst is a closed cavity with liquid or semi solid content with an epithelial lining. Childhood cystic masses can be difficult to characterise when they are huge and occupy most of the abdomen. The cyst may distort normal anatomy and their site of origin may be difficult to ascertain. The mass may be discovered late due to absence of early physical signs. In a female neonate, differential diagnosis includes ovarian cyst, sacrococcygeal teratoma (SCT), meningocele and a host of other tumours. We report a case of a -9- day old term female with pelvic cystic mass who presented with urinary retention. Urinary symptoms are very rare in SCT and are reported in the literature as a few sporadic cases. The tumour was resected and subsequently she no longer developed urinary retention and able to pass urine normally.

\section{Case report}

A termed baby girl weighing $3.9 \mathrm{~kg}$ presented at day 6 of life with fever, straining and poor urine output. She was noted to have a lower abdominal mass by a private paediatrician. At the private centre, an ultrasound scan was done. It showed a cystic mass in the pelvis separated from the urinary bladder. She also had a CT scan done which demonstrated a cystic lesion arising from the pelvic cavity extending into the lower abdomen measuring $6.4 \mathrm{~cm} \times 3.2 \mathrm{~cm} \times 3.7 \mathrm{~cm}$ with probability of a large ovarian cyst. A urinary catheter had to be inserted to relieve her symptom. She was referred to our unit at day 19 of life following recurring episodes of urinary retention each time the catheter was taken off.

Our assessment revealed a healthy baby girl with a large fixed supra pubic mass which was tender to palpation. The urinary catheter was in situ and draining clear urine. Per rectal examination was done, the mass was easily palpable at pre sacral area. An urgent ultrasound abdomen was performed; it revealed a cystic mass in the pelvis $10 \mathrm{~cm} \times 5 \mathrm{~cm}$; (larger than previously documented radiologically), no calcification was noted, there was also mild right hydronephrosis. An MRI was carried out to enable us to differentiate between anterior meningocoele and a sacrococcygeal teratoma. The MRI showed a large pre-sacral cystic mass consistent with an intra-pelvic teratoma (Figure 1). Her Alpha-fetoprotein (AFP) was 3746 unit (normal range
$0.00-6.74$ ). BHCG $4.2 \mathrm{IU} / \mathrm{L}$ (normal range $<5 \mathrm{IU} / \mathrm{L}$ ). Her renal profile was also normal.

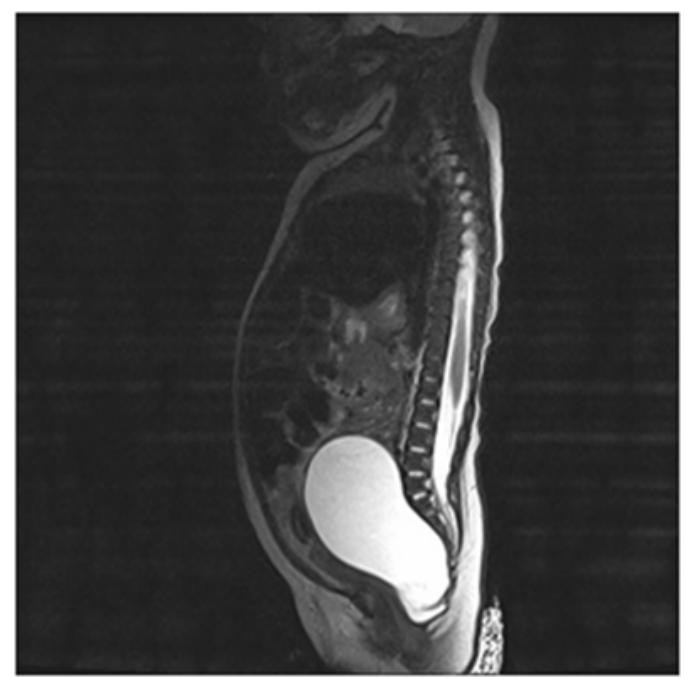

Figure I MRI Sagittal view; Arrow points to the pre-sacral cystic mass extending into the abdomen.

She subsequently underwent an elective laparotomy and excision of the tumour via a pfannensteil incision. The pre-sacral mass was predominantly cystic, approximately $10 \mathrm{~cm}$ X $10 \mathrm{~cm}$ in size. The cyst was drained to facilitate excision. Coccygectomy was also performed as part of a routine procedure for SCT operation. On palpation, the urinary bladder wall was thickened and large in size; rising suspicion of neurogenic bladder. There was no immediate post operative complication. She was discharged well on post-op day7 with the catheter left in situ. The urinary catheter was removed after 3 weeks. The patient managed to pass urine normally. Patient was closely followed up at our surgical clinic.

The histological findings of the lesion revealed a mature cystic teratoma composed of skin adnexa, hair follicle, and sebaceous, seromucous glands and adipocyted, glial and nerve bundles. Histology of the coccyx was confirmed free from teratomatous deposits. Serial alpha fetoprotein was normal at post-surgery one month, 3months, 
6months as well as annually for subsequent five years. She has normal milestone development and her micturition is normal and she is fully continent.

\section{Discussion}

Sacrococcygeal teratoma (SCT) is uncommon; however SCT is the commonest tumour in neonatal period. The incidence of the teratoma is 1 in 35000 to 40000 live births. ${ }^{1}$ SCT has a 4 to 1 female to male ratio. However it has been reported that in India the distribution is equal for both gender. ${ }^{1}$ The American Academy of Paediatrics surgical section classification helps to distinguish the locations of the SCT. ${ }^{2}$

i. Type I almost entirely external with minimal extension into pelvis.

ii. Type II mainly external with significant pelvic extension.

iii. Type III Majority is both external and internal with predominantly internal.

iv. Type IV completely internal, not visible externally.

The diagnosis for SCT can be detected antenatally by ultrasound scan. The gestation age for the detection of antenatal SCT ranged from 21 to 35 weeks. ${ }^{3,4}$ Antenatal ultrasound detection is associated with higher than expected incidence of perinatal and prenatal complications. Foetus with SCT that survives the gestation period and delivery tend to have good prognosis with early surgical intervention.

Our patient has type IV SCT diagnosed neonatally. Literature has reported that type IV completely cystic SCT is uncommon and occurs in about $15 \%$ of SCT. Urinary symptoms are rare and usually its presence is associated with malignant lesion. In our case, the tumour was huge and cystic. It displaced the rectum and uterus anteriorly and the bowels superiorly. There was also a mild right hydronephrosis. Literature review showed that there was only one reported case which was similar to ours, in 2005, however the long term follow-up was unknown. When urinary retention develops in neonate, a cystic abdomino-pelvic mass would be one of the differential diagnosis. ${ }^{5}$

Surgical resection remains the curative form of therapy for benign tumours; the gold standard has always been excision of tumour with removal of coccyx. ${ }^{6}$ Total excision of the tumour must include removal of the coccyx to prevent recurrence. ${ }^{7}$ Incomplete excision, incision and drainage of tumour is associated with risk of recurrence and malignant change. ${ }^{8}$ The probability of recurrence of tumour is high in the first 2 years after the initial surgery. ${ }^{9}$ The recurrence rate is between $3-35 \%$. ${ }^{1,6}$

\section{Conclusion}

$\mathrm{SCT}$, although histologically benign, has an alarming potential to recur either as a benign or malignant tumour during the first 2 years of life. Close follow-up for at least 3years (frequent examination, serial serum alpha-fetoprotein as well as diagnostic imaging if there raise serum alpha fetoprotein) is recommended for all patients who had undergone excision of SCT in the newborn period. ${ }^{10}$

Our patient has been on our follow up for over five years. There were no symptoms of urinary retention or signs of any tumour regrowth. Serial ultrasound scan, alpha fetoprotein level and clinical examination have not shown any sign of recurrence.

\section{Acknowledgments}

None.

\section{Conflicts of interest}

Author declares there are no conflicts of interest.

\section{Funding}

None.

\section{References}

1. A Wakhlu, S Misra, RK Tandon, et al. Sacrococcygeal teratoma. Pediatr Surg Int. 2002;18(5-6):384-387.

2. Altman RP, Randolph JG, Lilly JR. Sacrococcygeal teratoma: American Academy of Pediatrics Surgical Section Survey-1973. J Pediatr Surg. 1974;9(3):389-398.

3. Grisoni ER, Gauderer MWL, Wolfson RN, et al. Antenatal diagnosis of sacrococcygeal teratoma: prognostic features Pediatr Surg Int. 1998;3(2):173-175

4. Burgess I, Hines B, Stevenson P. Cystic Type IV sacrococcygeal teratoma detected at 18 weeks prenatal ultrasound. Ultrasound in Obstetrics and Gynecology. 2002;11(4):305.

5. Galili O, Mogilner J. Type IV sacrococcygeal teratoma causing urinary retention: a rare presentation. J Pediatr Surg. 2005;40(2):E18-E20.

6. De Backer A, Hazebroek F. Reoperative Pediatric Surgery. Caniano, D. Teich, S. (eds.). Humana Press, (Reoperative Pediatric Surgery). 2009;29:467-474.

7. Whalen TV, Mahour GH, Landing BH, et al. Sacrococcygeal teratomas in infants andchildren. Am J Surg. 1985;150(3):373-375.

8. Singhal GD, Gopal SC, Gangopadhay AN. Sacrococcygeal teratoma: an analysis of 37 cases. Indian J Pediatr. 1986;53(1):115-118.

9. Schmidt B, Haberlik A, Uray E, et al. Sacrococcygeal teratoma: clinical course and prognosis with a special view to long-term functional results. Pediatr Surg Int . 1999;15(8):573-576.

10. Bilik R, Shandling B, Pope M, et al. Malignant benign neonatal sacrococcygeal teratoma. J Pediatr Surg. 1993;28(9):1158-1160. 